\title{
CAD techniques for microwave circuits
}

\section{by I. D. Robertson, U. Karacaoglu and D. Sánchez-Hernández}

In little more than 10 years computer-aided design (CAD) of microwave circuits has moved from dumb terminals on mainframe computers to PCs, and now to powerful

RISC workstations. Commercial CAD software now integrates the various stages of microwave circuit design: schematic capture, simulation and layout. This paper reviews the different CAD packages that are available for microwave circuit design. The basic principles employed in the modelling of microstrip circuits are introduced and the

reasons for the extensive use of frequency-domain simulations are explored. The developments in nonlinear, electromagnetic and system-level simulation methods are described.

\section{Introduction to microstrip modelling}

There are several transmission-line media available for the microwave designer nowadays ${ }^{1}$ (microstrip, stripline, coplanar waveguide, thin-film microstrip, suspended microstrip, etc.), but microstrip transmission lines remain the most popular choice for realising microwave integrated circuits and even MMICs (monolithic microwave integrated circuits). ${ }^{2}$ Microstrip circuits can easily be fabricated by photolithographic techniques and components such as transistors can readily be soldered or bonded onto them. In order to introduce the principles of microstrip circuit modelling the lowpass filter shown in Fig. 1 serves as a convenient design example. This filter employs wide line sections as shunt capacitance elements and narrow line sections as series inductance elements. For the first stage of the design process the microstrip lines are all treated as ideal transmission lines with a certain characteristic impedance and electrical length. The minimum and maximum widths of the lines are determined by the lowest and highest characteristic impedances that can practically be employed. The electrical length of each section can be calculated using well known synthesis techniques. ${ }^{3}$.

The second stage of the design procedure involves converting the ideal electrical parameters into physical parameters, i.e. the width and length of each section. This is not straightforward because microstrip does not support pure TEM propagation (transversal electromagnetic propagation, where both electric and magnetic field components are perpendicular to the direction of propagation) and there are no exact analytical expressions for the calculation of widths and lengths. Instead, there are well known closed-form expressions which are used in calculating the physical parameters. ${ }^{4-5}$ These are derived using conformal mapping techniques based on a quasi-
TEM approximation and are at the heart of microstrip CAD programs such as Touchstone ${ }^{\mathrm{TM}}$ and tools such as Linecalc ${ }^{\mathrm{TM}}$. Whether these formulas are accurate depends on the substrate thickness, its dielectric constant and the operating frequency. At higher frequencies the microstrip line is able to support more than one mode of propagation and the assumption of quasi-TEM behaviour may become

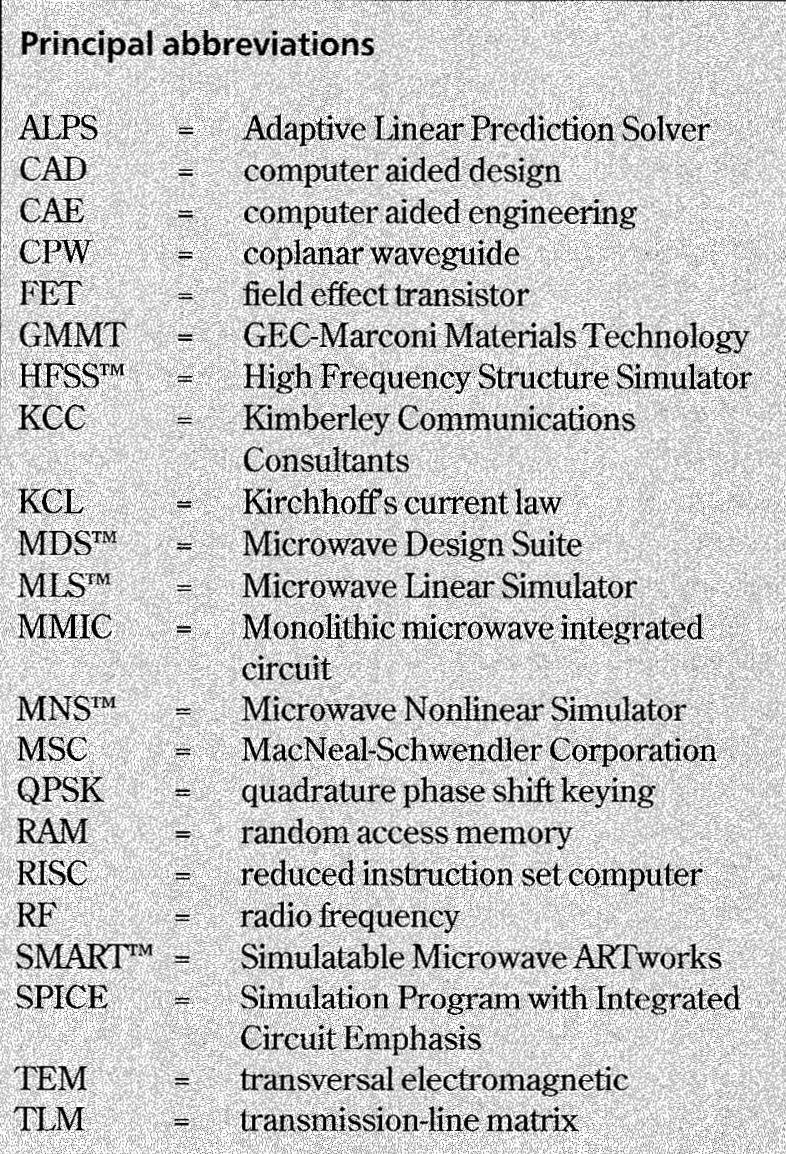


completely inapplicable. Even when the fields are quasiTEM, there is a dispersion effect because the fields are partly in the dielectric material and partly in air: as the signal frequency goes up the field pattern alters and the characteristic impedance and effective dielectric constant change. A further complication in making the conversion to a physical layout is the presence of discontinuities. In the lowpass filter example the only discontinuities are the steps in width. In general there may be a range of discontinuities, such as T-junctions, bends, open-circuited ends, curves, tapers and crosses. At microwave frequencies these discontinuities have a major effect on the circuit and must be incorporated into the circuit simulation. The discontinuities are usually incorporated into the circuit simulation by the use of equivalent circuit models.

This simple example illustrates that when designing microstrip circuits it is often observed that the actual performance significantly differs from the theoretical calculations. This is mostly due to factors such as dispersion, attenuation or discontinuity effects, which are functions of physical parameters and the circuit layout. To minimise the need for successive iterations, the designer relies on $\mathrm{CAD}$ techniques. $\mathrm{CAD} / \mathrm{CAE}$ systems allow design to be performed more quickly and accurately than would be possible with the old manual methods and the CAD program can readily create layout files for mask generation and circuit fabrication. Synthesis tools such as $\mathrm{E}-S y n^{\mathrm{TM}}$ and Matchnet ${ }^{\mathrm{TM}}$ help the designer carry out the electrical design of filters and matching networks using established synthesis techniques. Programs such as Touchstone are then used to simulate the performance of the circuit, which is defined entirely in terms of its physical layout dimensions. This layout-riented approach is a key difference between microwave $\mathrm{CAD}$ and conventional circuit simulators like SPICE. ${ }^{6}$

\section{Linear frequency-domain analysis}

The second key difference between microwave CAD and SPICE-like circuit simulators is that microwave circuit analysis is largely performed in the frequency domain. Working in the frequency domain means that only the steady-state response of distributed elements (transmission lines) needs to be considered. This is very important, because the multiple reflections encountered in a microwave circuit would take a lot of time to solve in the time domain. Furthermore, the frequency dependence of transmission-line parameters, resulting from dispersion, cannot be handled easily in the time domain.

Linear frequency-domain analysis gives a timeindependent solution for linear networks with sinusoidal excitation at a range of frequencies. Nonlinear components are treated with linearised small-signal models: a component's complex behaviour is simplified by assuming that the AC signal is causing only small perturbations around the DC operating point. Small-signal linear simulation can also take into consideration temperaturedependent thermal noise from lossy passive elements and temperature-and bias-dependent noise from active devices and sources. This type of simulator generally uses

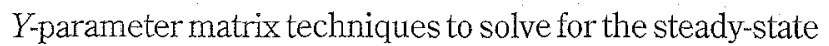
frequency response; Touchstone is the most familiar example. Fig. 2 shows a screen dump of the lowpass filter in Fig. 1 entered into Touchstone for Windows. The three windows show the network listing, the simulated results and the autoprocessed layout view (where the layout is obtained by directly processing the network list).

In the network listing approach, the circuit network is described by a list of components which are interconnected by a series of nodes, also described in the list. In this approach the designer has to remember, or constantly look up, the syntax for the component being included in the circuit. This disadvantage is overcome by schematic capture, in which a schematic symbol is placed on the working page and the designer is prompted for the relevant parameters that are needed to fully describe the component, as will be described later.

Since individual components are treated essentially as frequency-dependent admittances within a nodal matrix, solution for the overall circuit response is a case of using matrix reduction techniques to end up with an overall set of Y-parameters (admittance parameters). These can then be converted to $S$-parameters (scattering parameters), etc. for display. $S$-parameters are universally used by microwave engineers since they are ideally suited to the understanding of transmission-line circuits. The simulator does not need to calculate the currents or voltages in the circuit and the analysis is very fast as a result. However, the nonlinear large-signal operation cannot be modelled because all the elements are defined by linear models.

\section{Microstrip discontinuity models}

Most models are based on published information (quasistatic models), which is then somehow modified for the specific package. As examples, the step, $90^{\circ}$ mitred bend and T-junction schematic elements in Libra ${ }^{\mathrm{TM}}$ are shown in Fig. 3. The models follow the straight resonator method $^{7}$ and the empirically based, analytical model which consists of a static, lumped, equivalent circuit. The lumped-element equivalent circuit models can also be observed in Fig. 3. For the chamfered microstrip bend, the equivalent circuit parameters are calculated based on the expressions in Reference 8 , which are reproduced here to illustrate the empirical nature of the models:

$$
\begin{aligned}
& \frac{C}{H}=\frac{W}{H}\left[7 \cdot 6 \varepsilon_{r}+3 \cdot 8+\frac{W}{H}+\left(3.93 \varepsilon_{r}+0.62\right)\right] \quad \mathrm{pF} / \mathrm{m} \\
& \frac{L}{H}=441.2712\left[1-1.062 \exp \left(-0.177\left(\frac{W}{H}\right)^{0.947}\right)\right] \mathrm{nH} / \mathrm{m}
\end{aligned}
$$

where $C$ and $L$ are the capacitance and inductance of the lumped element model of Fig. 3 and $W$ and $H$ are the conductor width and the substrate thickness, respectively. The frequency-domain analytical model on which the step in width simulation is based is derived from a TEM planar waveguide model of the discontinuity. The waveguide model was then transformed into a rectangular waveguide 


\section{CAD}

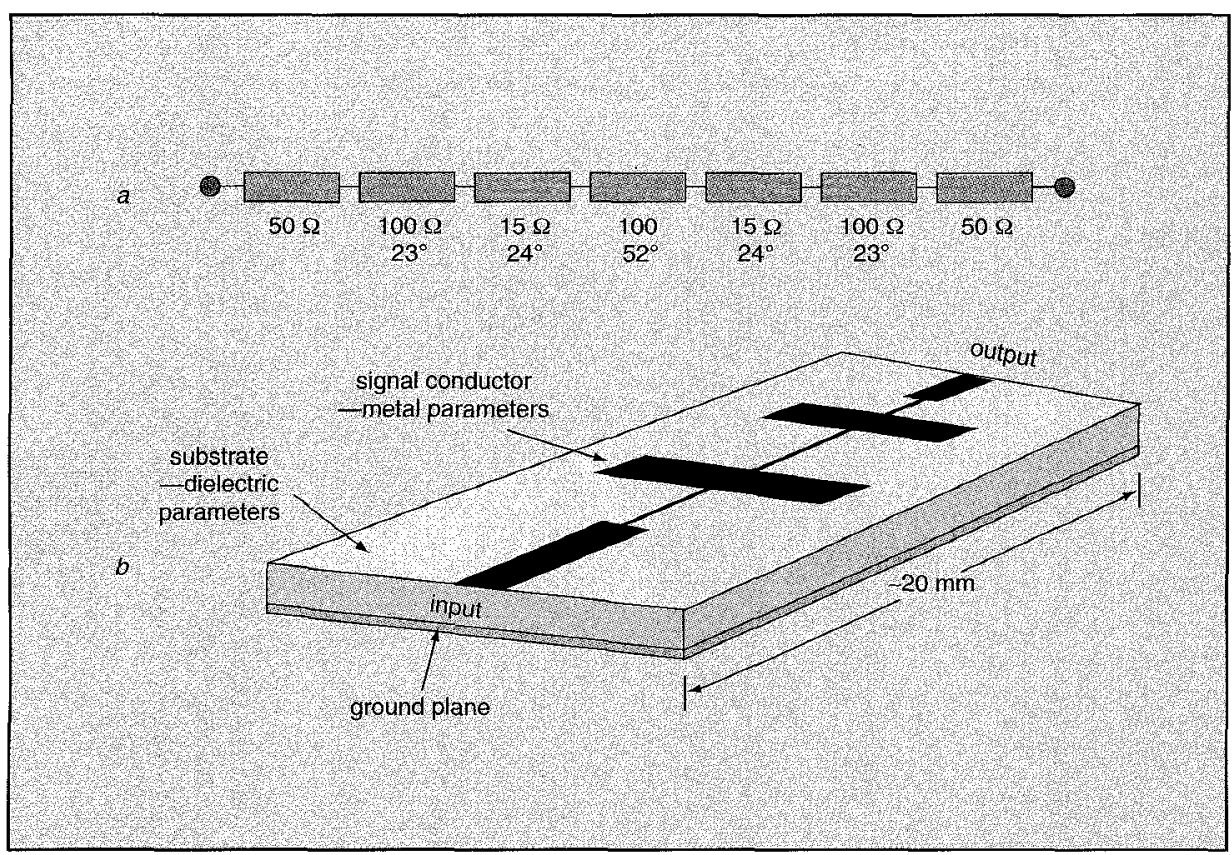

Fig. 1 Microstrip lowpass

filter: (a) synthesised electrical circuit with characteristic impedances and electrical lengths in degrees at $4 \mathrm{GHz}$;

(b) physical realisation

model and the expression for the series inductance $L_{s}$ in Fig. $3 a$ was formulated based on an analysis of the current concentration at the discontinuity. ${ }^{10}$ In addition, dispersion is accounted for in the model.

The T-junction model is based on Hammerstad's discontinuity mode ${ }^{11}$ and a reference plane shift is added to each port to make the reference planes consistent with the layout. Dispersion is accounted for in both the reference plane shifts and the shunt susceptance calculations using the formulas in Reference 8 . The validity of the models is often constrained by conservative design limits, also given in Fig. 3. These limits indicate a range of parameter values within which the model is known to be valid in practical applications. If only approximate results are required, this range may be much greater. Additionally, for transient analyses, the accuracy of the model decreases for $W / H<0.32$, due to the static and limited nature of the model.

\section{Schematic capture for microwave circuits}

Performing a detailed simulation of a microwave circuit is a step-by-step procedure. For example, the first task in designing a microwave amplifier is to find a robust circuit topology by modelling only the basic circuit, which may have been designed with a Smith chart. Only after the basic topology has been simulated are the effects of discontinuities considered. However, the inclusion of discontinuity models in the circuit then introduces additional nodes. With an ordinary network-list ('net-list') approach the circuit description quickly becomes unwieldy as new nodes are added and the node numbers get completely out of sequence. With a net-list approach the circuit diagram has to be drawn manually on paper from time to time to check that the nodes are still correct. Mistakes can easily occur with this manual procedure.

This problem is overcome with schematic capture, which is very convenient for microwave circuits. Here, the design is entered graphically as a circuit diagram using symbols for the microstrip elements. Entry of the component parameters is usually through a series of popup windows that prompt for each parameter required. When all the appropriate entries have been made, the designer closes the window and the component will be placed on the working page and the schematic symbol shown with the associated component values. To avoid unnecessary clutter on the working page, the display of these parameters can often be hidden, although the information is of course still available in the simulator's design database.

For this method to be of any value, there must be schematic symbols associated with every component that can be placed in the design file. This is now a fundamental part of most of the major CAD packages and the old net-list is often not accessible. The advantage of schematic capture is that it is easy to visualise your circuit and prevent mistakes. To achieve this, designs should use hierarchy

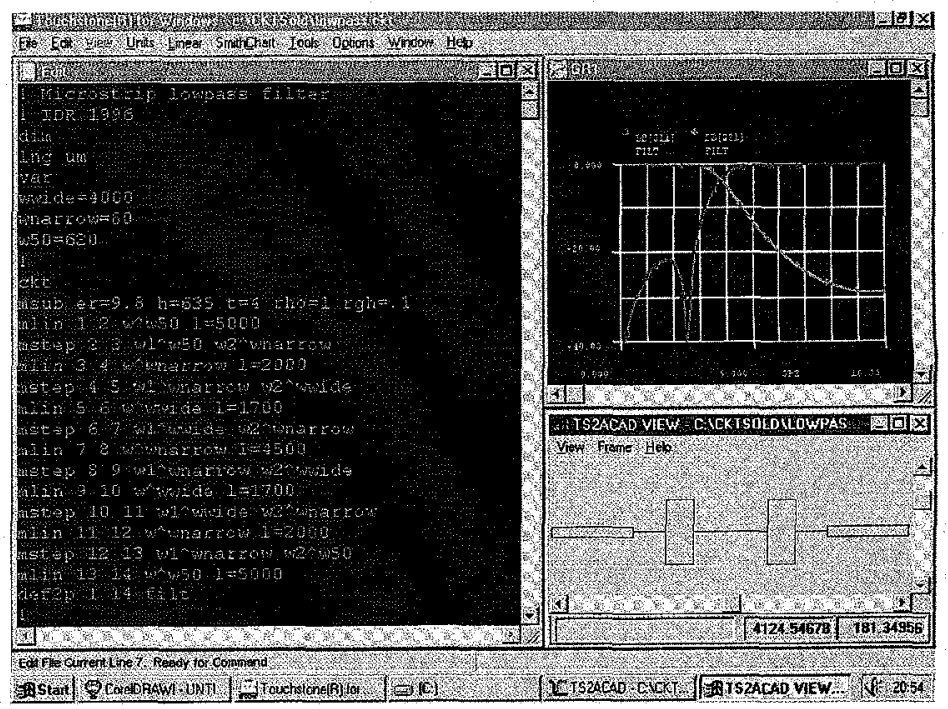

Fig. 2 Microstrip lowpass filter in Touchstone for Windows 


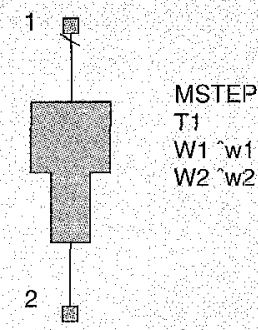

Range of usage

$-01 \leq \frac{W 2}{W 1} \leq 10$

- $\varepsilon_{r} \leq 10$

a MSTEP

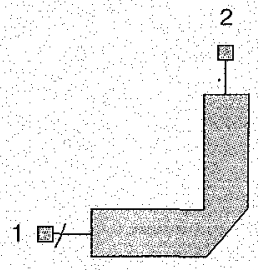

MBEND BEND1

$W=25$

$A N G=90$

$\mathrm{M}=0.60$

Range of usage

$\bullet 0.5 \leq \frac{W}{H} \leq 2.75$

- $2 \cdot 75 \leq \varepsilon_{r} \leq 25$

- simu treq $=\frac{15}{H(\mathrm{~mm})} \mathrm{GHz}$

$b$ MBEND
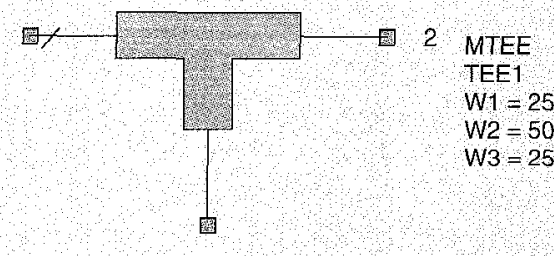

3

Range of usage

- $w 1+w_{3} \leq 0.5 \lambda$

- $W 2+W 3 \leq 0.5 \lambda$

- $011 H s W_{1}, W_{2}, W_{3} \leq 10 H$

- $\varepsilon_{r} \leq 128$

Equivalent circuit

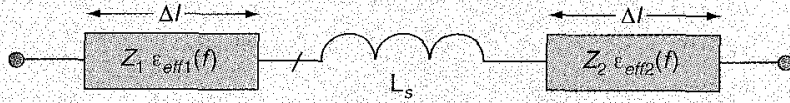

$L_{s}$

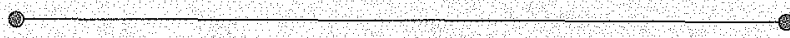

Equivalent crrcuit

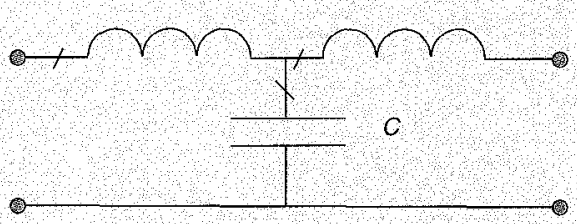

W $3=25$

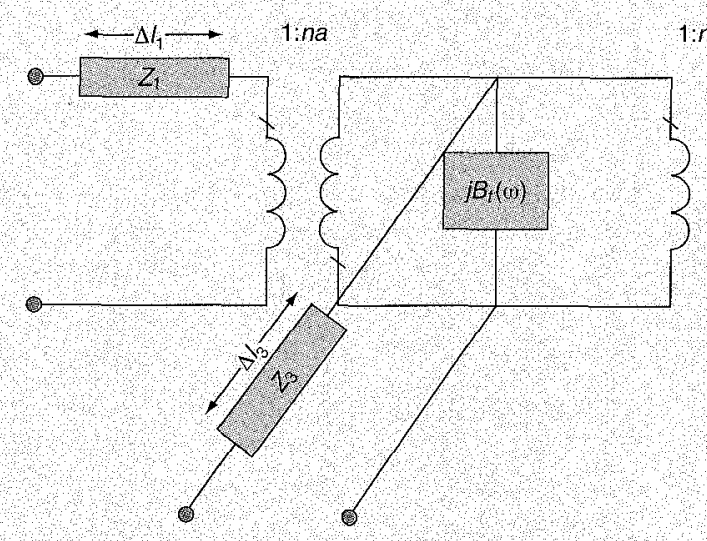

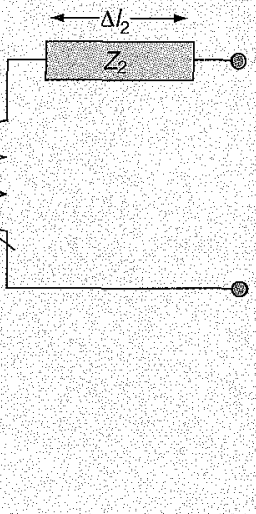

C MTEE

Fig. 3 Microstrip elements on Libra Series IV: (a) step; (b) $90^{\circ}$ mitred bends; (c) T-junction

extensively in order to keep them tidy and easy to follow. Fig. 4 shows the Libra schematic of the lowpass filter; each $\mathrm{CAD}$ element has its own symbol and these are wired together on the circuit page.

\section{Commercial microwave CAD packages}

The special requirements for microstrip circuit modelling were first met by programs such as Compact ${ }^{\mathrm{TM}}$, and later by Super-Compact ${ }^{\mathrm{TM}}$ and Touchstone. The main limitations of these types of simulators are their limited accuracy, especially at high microwave frequencies, and their inability to treat nonlinear circuits. As a result, there has been a rapid development of other CAD techniques, especially for electromagnetic simulation and for nonlinear circuit modelling. Electromagnetic simulation is important for microwave circuits because the approximate models used in conventional simulators assume quasi-TEM behaviour and assume that individual discontinuities and lines do not interact. Electromagnetic simulators can look at the whole picture, rather than breaking the circuit down into building blocks, and can conduct a full-wave analysis by solving Maxwell's equations for two-dimensional and threedimensional transmission-line problems. There is now a large range of commercially available microwave $C A D$ packages. The key players in the major microwave CAD business are listed in Table 1. The most notable development in this area recently was the acquisition of EEsof (who first 
developed Touchstone and its related products) by Hewlett Packard (who already had their own $\mathrm{MDS}^{\mathrm{TM}}$ suite). A unified CAD package using the best elements of the HP and EEsof products is eagerly awaited. With such a wide range of software on offer, it is important to understand the features and limitations of each of the different types of microwave simulator. To this end, there follows a description of the salient features of these different simulation techniques.

\section{Nonlinear modelling}

The basic design of an amplifier can be carried out entirely using linear simulations based on the transistor's measured $S$-parameters or a small-signal equivalent circuit model. However, often the designer needs to be able to predict and optimise the amplifier's output power and intermodulation performance. Other circuits which require large-signal simulations are mixers and oscillators. In these cases, nonlinear simulation is necessary, but using ordinary SPICE means that the required microstrip library elements are not available. In addition, there are particular difficulties attached to using time-domain simulation for microwave circuits: firstly, distributed circuits can take a long time to reach a steady state, and, secondly, microstrip lines are dispersive, with frequency-dependent behaviour. As a result, special microwave nonlinear simulators have been developed which use either a convolution technique or the harmonic-balance method.

\section{Time-domain simulation}

To calculate the true response of nonlinear components such as transistors it is necessary to work in the time domain. This is because the equivalent circuit elements vary with signal amplitude and are thus functions of time. Direct time-domain methods are generally based on SPICE, with more convenient display options added to create programmes such as MicroWave-Spice ${ }^{\mathrm{rM}}$ (now known as the Time Domain Test Bench in Series IV). SPICE models a circuit by representing the differential equations whereby each component is modelled in terms of finite difference equations (such as $i=C d v / d t$ for a capacitor) and solving the set of nonlinear algebraic functions at each time sample in an iterative manner. A set of initial conditions has to be imposed on the network to

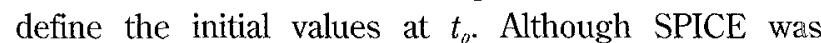
conceived to analyse transient effects in a circuit, it can also analyse steady-state operation by simply extending its transient analysis out to steady-state. Given that the time step has to be chosen small enough to achieve accuracy and prevent divergence, this can be a big drawback for microwave circuits, especially when the transients are not speedily dispersed (high- $Q$ circuits and, in general, circuits where the time constants are much longer than the period of excitation). Besides, for multitone excitation the steadystate condition is difficult to determine and only relatively low dynamic range can be achieved.

\section{Convolution simulation}

The need to consider losses, multitone excitation, dispersion for distributed elements and parasitics makes the use of conventional time-domain simulators inefficient. Yet, some of the newer time-domain simulators use a convolution technique which does make it possible to handle dispersion (e.g. Impulse ${ }^{\mathrm{TM}}$ ). The frequency-domain information of all the distributed elements is converted into the time-domain, effectively resulting in the impulse response of these elements.

The time-domain input signal at the element terminals is then convolved with the impulse-response of the element to obtain the output signal. Those elements that have exact lumped equivalent models are characterised in the time domain. As a result, the models in HP Impulse accurately

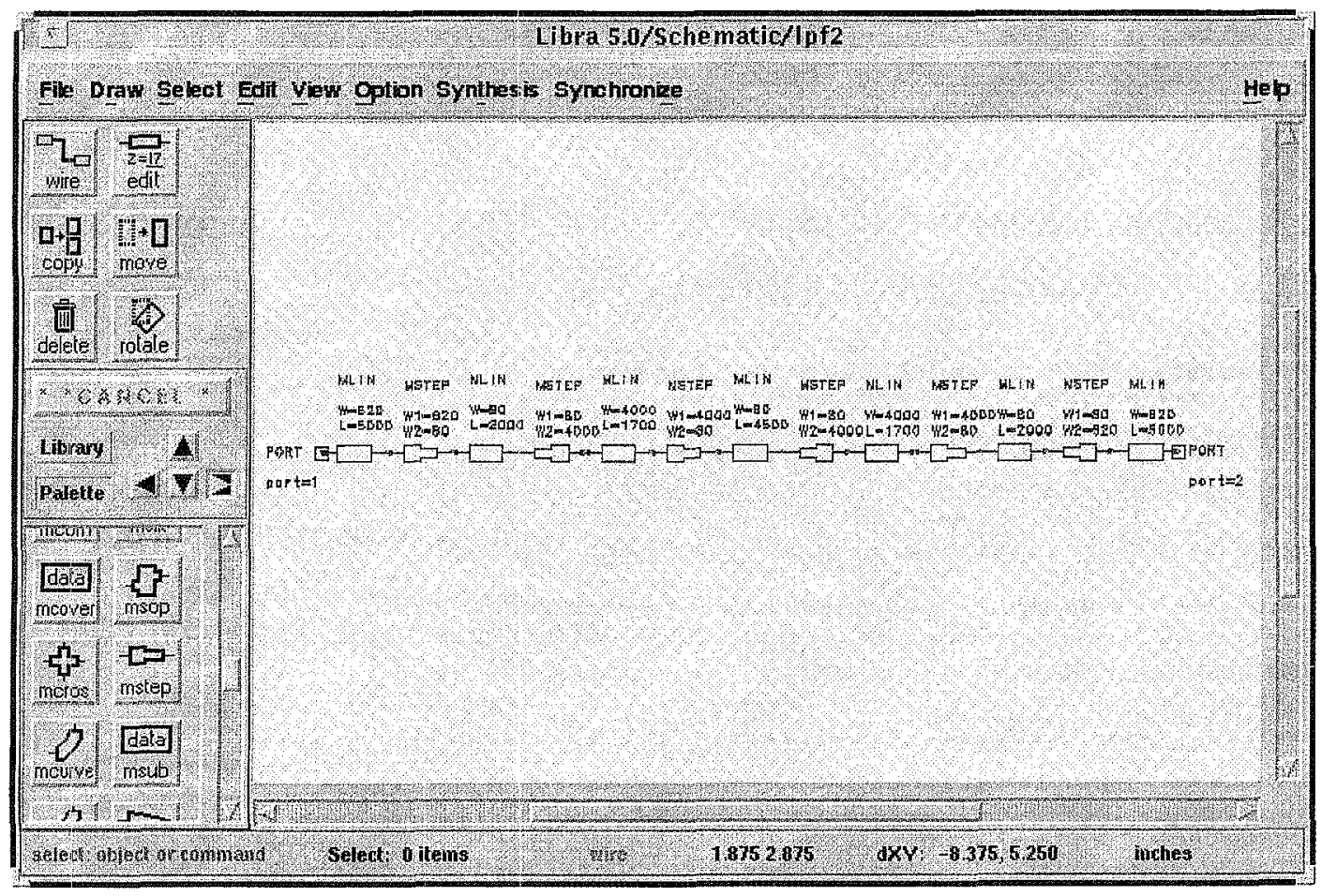

Fig. 4 Libra schematic diagram of the lowpass filter 


\section{CAD}

Table 1: Key players in the microwave CAD business and their products

\begin{tabular}{|c|c|c|}
\hline Company & $\begin{array}{l}\text { Product } \\
\text { (all trademarks) }\end{array}$ & Type \\
\hline HP-EEsof (HP range) & $\begin{array}{l}\text { MDS } \\
\text { MLS } \\
\text { MNS } \\
\text { Impulse } \\
\text { Momentum } \\
\text { HFSS } \\
\text { MW Artwork Generator }\end{array}$ & $\begin{array}{l}\text { Integrated package } \\
\text { Linear simulator } \\
\text { Harmonic balance } \\
\text { Time domain } \\
\text { 3-D planar electromagnetic } \\
\text { 3-D arbitrary electromagnetic } \\
\text { Layout }\end{array}$ \\
\hline HP-EEsof (EEsof range) & $\begin{array}{l}\text { Touchstone } \\
\text { Linecalc } \\
\text { Libra } \\
\text { MicroWave Spice (now in Libra) } \\
\text { E-Syn } \\
\text { Communications Design Suite }\end{array}$ & $\begin{array}{l}\text { Linear } \\
\text { Tansmission line tool } \\
\text { Harmonic balance } \\
\text { Time domain } \\
\text { Filter synthesis } \\
\text { System simulation }\end{array}$ \\
\hline Compact Software & $\begin{array}{l}\text { EASI } \\
\text { Serenade } \\
\text { SuperCompact } \\
\text { Harmonica } \\
\text { SuperSpice } \\
\text { Success } \\
\text { Microwave Explorer }\end{array}$ & $\begin{array}{l}\text { Integrated suite } \\
\text { Schematic capture } \\
\text { Linear } \\
\text { Harmonic balance } \\
\text { Time domain } \\
\text { System simulation } \\
\text { 3-D planar electromagnetic }\end{array}$ \\
\hline Sonnet Software & $\begin{array}{l}\text { em } \\
\text { xgeom } \\
\text { emvu } \\
\text { patgenlpatvu }\end{array}$ & $\begin{array}{l}\text { 3-D planar electromagnetic } \\
\text { Layout entry } \\
\text { Current display } \\
\text { Patch antenna patterns }\end{array}$ \\
\hline Jansen Microwave & $\begin{array}{l}\text { Linmict } \\
\text { Limmict/N } \\
\text { Unisym/Sfpmic }\end{array}$ & $\begin{array}{l}\text { Linear (integrated suite) } \\
\text { Nonlinear } \\
\text { 3-D planar electromagnetic }\end{array}$ \\
\hline Ansoft Corporation & $\begin{array}{l}\text { Maxwell-Strata } \\
\text { Maxwell SI Eminence } \\
\text { Maxwell SI Spicelink }\end{array}$ & $\begin{array}{l}\text { 3-D planar electromagnetic } \\
\text { High-speed circuitry/EMV/EMC } \\
\text { High-speed digital design }\end{array}$ \\
\hline Argumens & $\begin{array}{l}\text { Octopus } \\
\text { Stingray } \\
\text { Shark }\end{array}$ & $\begin{array}{l}\text { Linear } \\
\text { 3-D planar electromagenetic } \\
\text { Layout }\end{array}$ \\
\hline Barnard Microsystems & WaveMaker & $\begin{array}{l}\text { Layout, linear simulation, schematic } \\
\text { capture, device modelling (modules) }\end{array}$ \\
\hline Zeland Software & IE 30 & 3-D planar electromagnetic \\
\hline UC Boulder & PMESH & $2 V_{2}$-D electromagnetic \\
\hline Optimization System Associates & $\begin{array}{l}\text { Hope } \\
\text { Harpe } \\
\text { Empipe } \\
\text { Empipe3D/EmpipeExpress }\end{array}$ & $\begin{array}{l}\text { Linear/nonlinear } \\
\text { Parameter extraction } \\
\text { Sonnet, Ansoft, HP optimisation } \\
\text { Geometry capture front-end }\end{array}$ \\
\hline MacNeal-Schwendler Corporation & MicroWaveLab & 3-D arbitrary electromagnetic \\
\hline Kimberley Communications Consultants & Micro-Stripes & $\begin{array}{l}\text { 3-D arbitrary } \\
\text { electromagentic (TLM) }\end{array}$ \\
\hline Boulder Microwave Technologies & Ensemble & 3-D planar electromagnetic \\
\hline Computer System Technologies & Mafia & 3-D planar electromagnetíc \\
\hline Optotek & MMICAD & Linear simulator \\
\hline
\end{tabular}


represent dispersive effects such as skin-effect loss and changes in propagation velocity and impedance with frequency.

\section{Harmonic-balance simulation}

For steady-state large-signal and repetitive (optimisation) analyses (such as gain compression of a power amplifier) the harmonic balance technique ${ }^{12}$ is faster than time-domain methods. This uses a combination of time- and frequency-domain analysis. The harmonic balance analysis can be performed in three ways. In the nodal form, ${ }^{13}$ the circuit unknowns are the voltages at every node in the circuit. This method has proved to be an efficient one when the number of nonlinear elements is large compared to the number of linear elements.

In the piecewise harmonic balance method, the circuit is segmented into a linear part and a nonlinear part connected to the linear part at a series of ports. The linear-nonlinear interface defines corresponding nodes. The voltages and

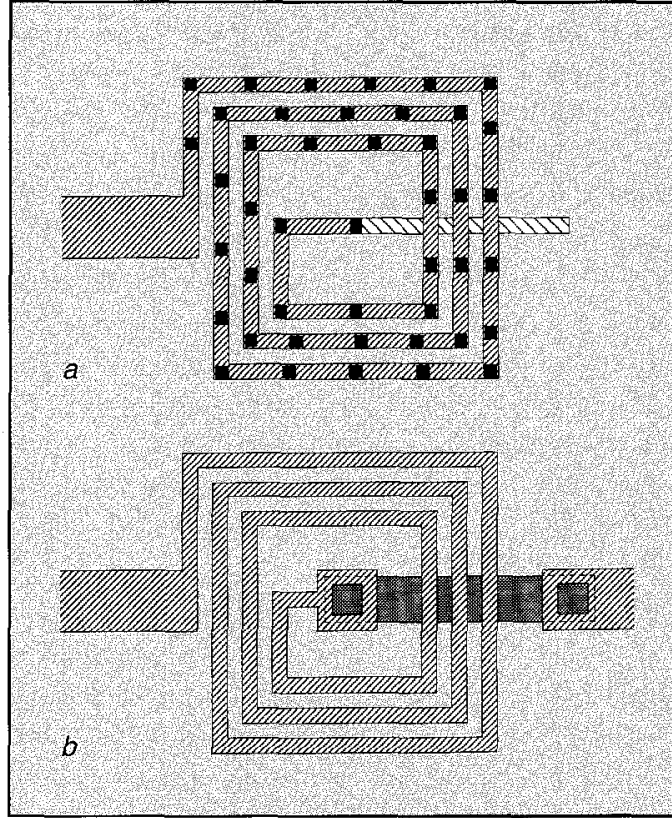

Fig. 5 MMIC spiral inductors: (a) using air bridges; (b) using two metal levels for an underpass
Examples of harmonic balance programs are Libra, Harmonica $^{\mathrm{TM}}$, and MNS $^{\mathrm{TM}}$ (Microwave Non-linear Simulator, within MDS). Currently, MDS appears to have the best harmonic-balance algorithm, which is able to handle three independent frequencies with 12 harmonics in the analysis. However, as much as 300 Mbytes of RAM may be required for such an analysis! currents in the nonlinear components are calculated in the time domain. The linear elements are treated in the frequency domain. Thus, every port is connected to a nonlinear device. Fourier transformation is then used to compare the currents and voltages of the nonlinear components with the terminating impedances presented by the linear components at all the harmonic frequencies used in the analysis. Any error is reduced by successive iterations.

The harmonic balance seeks a solution by matching the harmonic quantities at the linear-nonlinear interface to satisfy the continuity equation for current. Thus, in the harmonic balance method additional constraints (such as optimisation criteria) can be added into its system of equations. The signals must be periodic in order to use the Fourier transform. Due to the enormous number of calculations required and the need for iterations to ensure convergence, this type of analysis requires a fast computer with a lot of memory. These requirements go up dramatically as the number of harmonics increases.

An alternative to the previous two methods is the waveform balance method, ${ }^{14}$ where the response is represented in sampled data forms and the time-domain determining equations are satisfied for discrete time samples (time-domain harmonic balance where the unknowns are the samples of the waveforms themselves). The sensitivity between samples, however, is nonzero, making the waveform method practical only when the waveform is periodic. ${ }^{15}$

\section{MMIC modelling}

The major difference between modelling an ordinary microstrip circuit and modelling an MMIC is that the components on a given MMIC are specific to its particular manufacturer and the processing steps and materials used. For example, one manufacturer might use spiral inductors with airbridge crossovers, whereas another might use a dielectricspaced underpass to connect the centre turn back to the outside circuit, as shown in Fig. 5.

It is unreasonable to expect standard CAD packages to have library elements which cater for such a diverse range of passivê components. Instead, MMIC design relies largely on component models provided by the MMIC manufacturers themselves. Often, foundries supply a large number of $S$-parameter files to cover the most commonly used components. For example, data for 1 turn, 1.5 turn... up to 6 turn spiral inductors might be provided. If the measured data has been de-embedded accurately and the reference planes defined properly, then this data should be very reliable. However, it is often the case that, for example, a non-standard number of turns is required. This data-file approach has clear limitations for design and optimisation.

It is more common for a foundry to provide equivalent circuit models which are scalable in some way. In this approach a wide range of components are measured and an equivalent circuit model fitted to each one. Then, equations can be generated which give the values for the equivalent circuit as a function of either the layout dimensions (for a capacitor) or another parameter (such as the number of turns for an inductor). This approach has the advantage that the model is not limited to discrete values of standard elements. Optimisation can therefore be used. However, care must be exercised to ensure that the models are not used outside their range of validity and that they are accurate at high frequencies. The most common problems are encountered with lumped spiral inductor models, since these are often inaccurate past the self-resonant frequency of the inductor.

Active devices can be modelled with $S$-parameters, with small-signal equivalent circuit models, or with large-signal models. Many different large-signal models exist for field- 
effect transistors (FETs) such as the Curtice Cubic, Triquint's Own, HP Root, etc. The accuracy of most largesignal models still leaves a lot to be desired, especially for highly nonlinear circuits such as mixers.

\section{Simulator customisation: SMART ${ }^{T M}$ libraries}

In a fully integrated CAD package there is seamless integration of the schematic diagram and the layout. To achieve this level of design integration, the simulator must be customised to the foundry. This means that the foundry's models and layout library elements form a core part of the simulator's element library. The ability to edit the layout (i.e. the artwork) of a particular library element and then resimulate directly has led to the phrase Simulatable Microwave ARTworks, which was coined by EEsof. These SMART library elements have the component model and layout integrated, so the design becomes partially automated. It should be noted, however, that there is a limit to the complexity of a layout which can be created in this way. Apart from fairly simple circuits, most MMICs are too complicated to make full use of the automated layout facility. As an example, the Libra schematic of an MMIC amplifier using the GMMT SMART library set-up is shown in Fig. 6.

MMIC layout is still performed manually to a great extent. The best package for this is WaveMaker ${ }^{\mathrm{TM}}$ Layout, which offers a tremendous range of powerful layout commands yet is economical and can run on anything from a modest PC upwards. Fig. 7 shows a screen shot of an MMIC filter on WaveMaker. For the layout and designrule checking of complete mask-sets (i.e. a whole array of circuits, with alignment marks, etc.), powerful packages such as Cadence ${ }^{\mathrm{TM}}$ or Mentor-Graphics ${ }^{\mathrm{TM}}$ are important. Many microwave $\mathrm{CAD}$ companies now offer their simulator 'engines' for use with these industry standard interfaces.

\section{Electromagnetic simulation}

As has been mentioned before, electromagnetic simulators can be classified into two- or three-dimensional simulators. The two-dimensional field simulators are used to calculate the modal characteristics and electromagnetic fields for a cross-section of transmission lines embedded between layers of different dielectrics. The frequencydependent modal characteristics generated can be impedances, voltages, currents, powers, propagation velocities and effective dielectric constants. The threedimensional simulator can calculate the field pattern surrounding a general metal-dielectric structure problem. The two-dimensional field simulator restriction means that faster, more specialised methods can be used in the software. The result is that the time taken to achieve a solution is many orders of magnitude less than for the more general problem solvers. The three-dimensional former type of simulator relies on solution methods which divide the problem space into a point mesh and solve for the fields at the vertices.

\section{Planar electromagnetic}

These programs are intended to solve for the $S$ parameters of arbitrarily shaped microstrip or coplanar waveguide (CPW) structures. By limiting the problem to predominantly planar structures the speed of analysis is improved dramatically. Usually, the circuit conductors are divided into subsections and the method of moments is used for the electromagnetic analysis. Examples of this type of simulator are $\mathrm{em}^{\mathrm{TM}}$, Explorer ${ }^{\mathrm{TM}}$, and Momentum ${ }^{\mathrm{TM}}$. Often, many metal and dielectric layers can be handled, but these are assumed to be planar, and so the term 21/2-D has been coined (2-D currents but 3-D fields). These simulators cannot analyse true 3-D structures, such as microstrip-to-stripline transitions where the dielectrics are
Fig. 6 Libra schematic of MMIC amplifier using GMMTSMART library

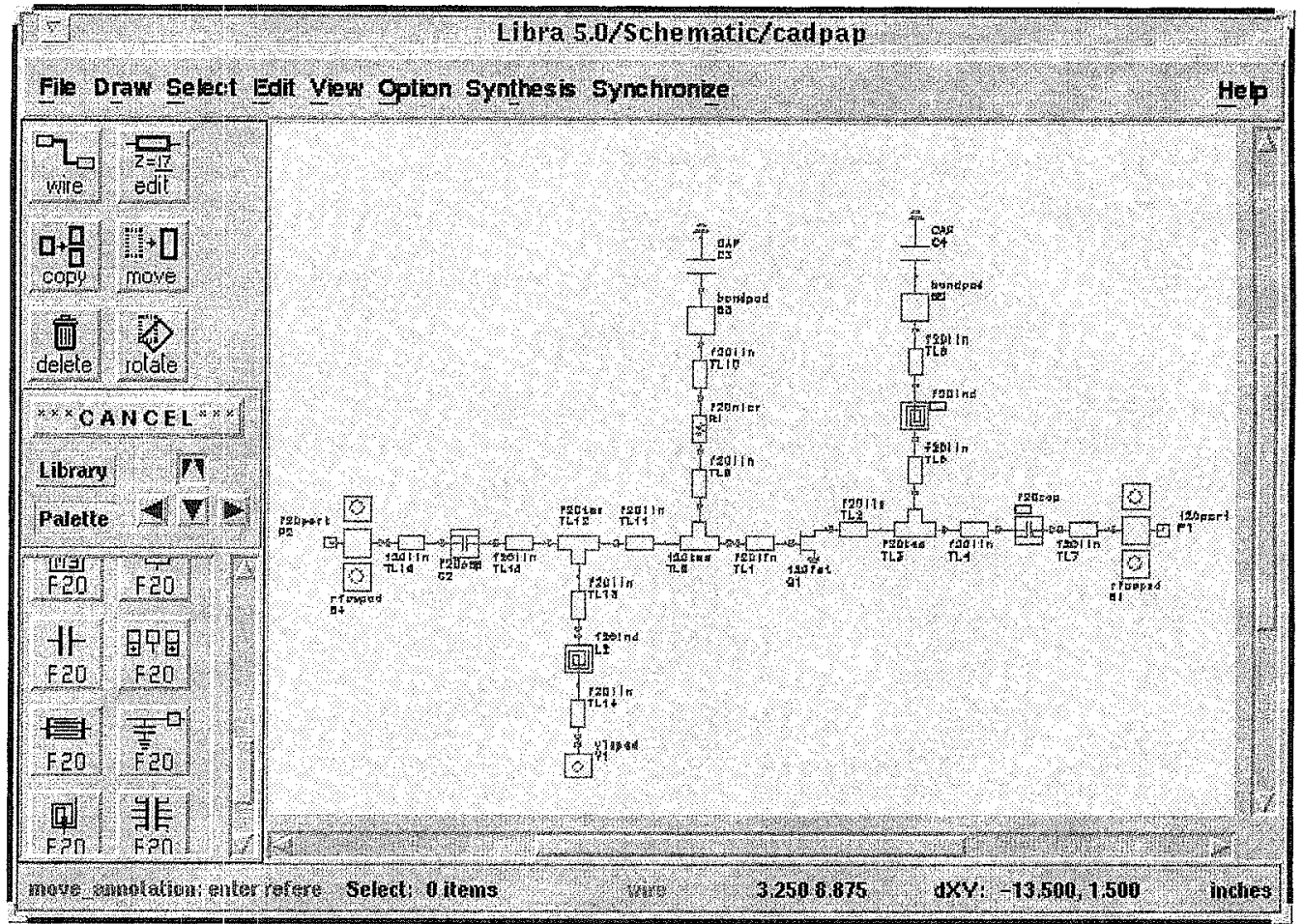


not planar. Yet, when the current on the third dimension can be used to represent, for example, vias, but the analysis allows only layered dielectrics, the term 3-D planar is more appropriate. ${ }^{16} 21 / 2-\mathrm{D}$ and 3-D planar simulators are used extensively in MMIC design to analyse non-standard microstrip structures such as coupled bends and meandered lines. Fig. 8 shows the layout of a meandered microstrip line on $x^{2}$ eom ${ }^{\mathrm{TM}}$ by Sonnet Software. The 3-D planar simulator em can handle any number of ports and metal or dielectric layers.

The speed of planar electromagnetic simulators makes them very practical for carefully investigating non-standard structures but conventional optimisation is not really feasible. For all these numerical methods the most timeconsuming step is the solving of the matrix; this involves a number $O\left(n^{3}\right)$ of operations, where $n$ is the number of elements in the matrix, while the building of the matrix encompasses $O\left(n^{2}\right)$ operations. Recently, however, a fast iterative matrix solver, with which solution time increases only as the square of the number of unknowns rather than the traditional cubic technique, has been developed by Ansoft Corporation for its 3-D planar Maxwell-Strata ${ }^{\mathrm{TM}}$ electromagnetic simulator. This method, coined ALPS, is an adaptive procedure that allows wideband $S$-parameter data and radiation to be computed from a handful of singlefrequency analyses at the dominant poles and zeros. OSA (Canada) has, however, made a breakthrough in electromagnetic optimisation with the development of the Space-Mapping $^{\text {TM }}$ optimisation technique ${ }^{17}$ used in the Empipe $^{\mathrm{TM}}$ family. For $3-\mathrm{D}$ planar electromagnetic simulators, at least $16 \mathrm{MB}$ of RAM is generally required, and at least $32 \mathrm{MB}$ swap space is needed in the hard disk. Additionally, around $100 \mathrm{MB}$ are needed to load and store the program.

\section{3-D electromagnetic}

The High Frequency Structure Simulator (HFSS ${ }^{\mathrm{TM}}$ ) by Hewlett Packard (3-D arbitrary) has dominated this market for several years. This uses finite-element techniques to analyse completely arbitrary metal and dielectric structures and can thus be used for analysing components such as waveguide-tomicrostrip transitions and MMIC packages. However, this type of analysis is extremely demanding of CPU time and memory: Hewlett Packard currently recommends around 300 Mbytes of RAM.

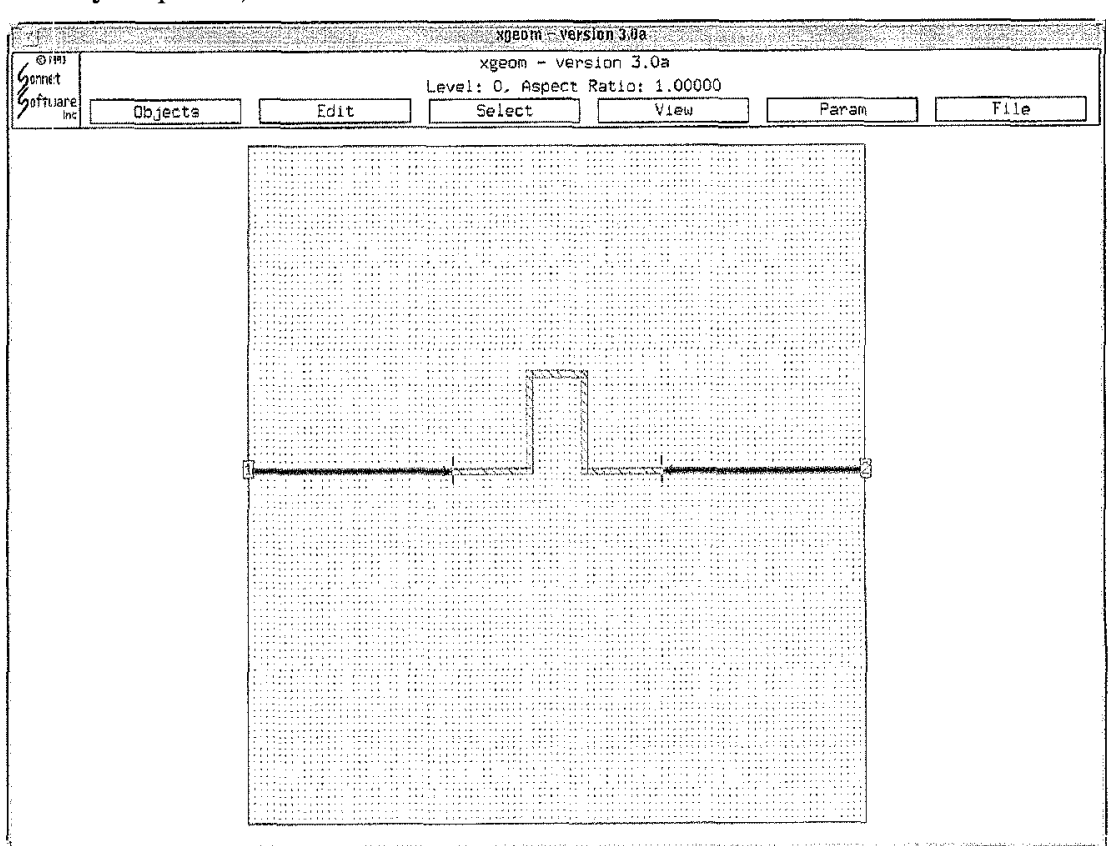

Fig. 8 Meandered line on $x g e o m$
More recently, some other companies have introduced 3-D simulators: for example the MacNeal-Schwendler Corporation (MSC) has introduced 'MicroWave Lab' and Kimberley Communications Consultants (KCC) has introduced 'Micro-Stripes'. Micro-Stripes ${ }^{\mathrm{TM}}$ uses the transmission-line matrix (TLM) method, which is a fast technique requiring less memory than finite-element methods. The TLM method simulates the propagation of electromagnetic waves in any medium where both space and time are discretised. The space is modelled by a transmission-line-bounded network which allows the description of 3-D structures made up of dielectric substrates and metallic strips with finite dimensions.

\section{Time-domain versus frequency-domain}

Whether the 3-D simulator should be based on time- or 


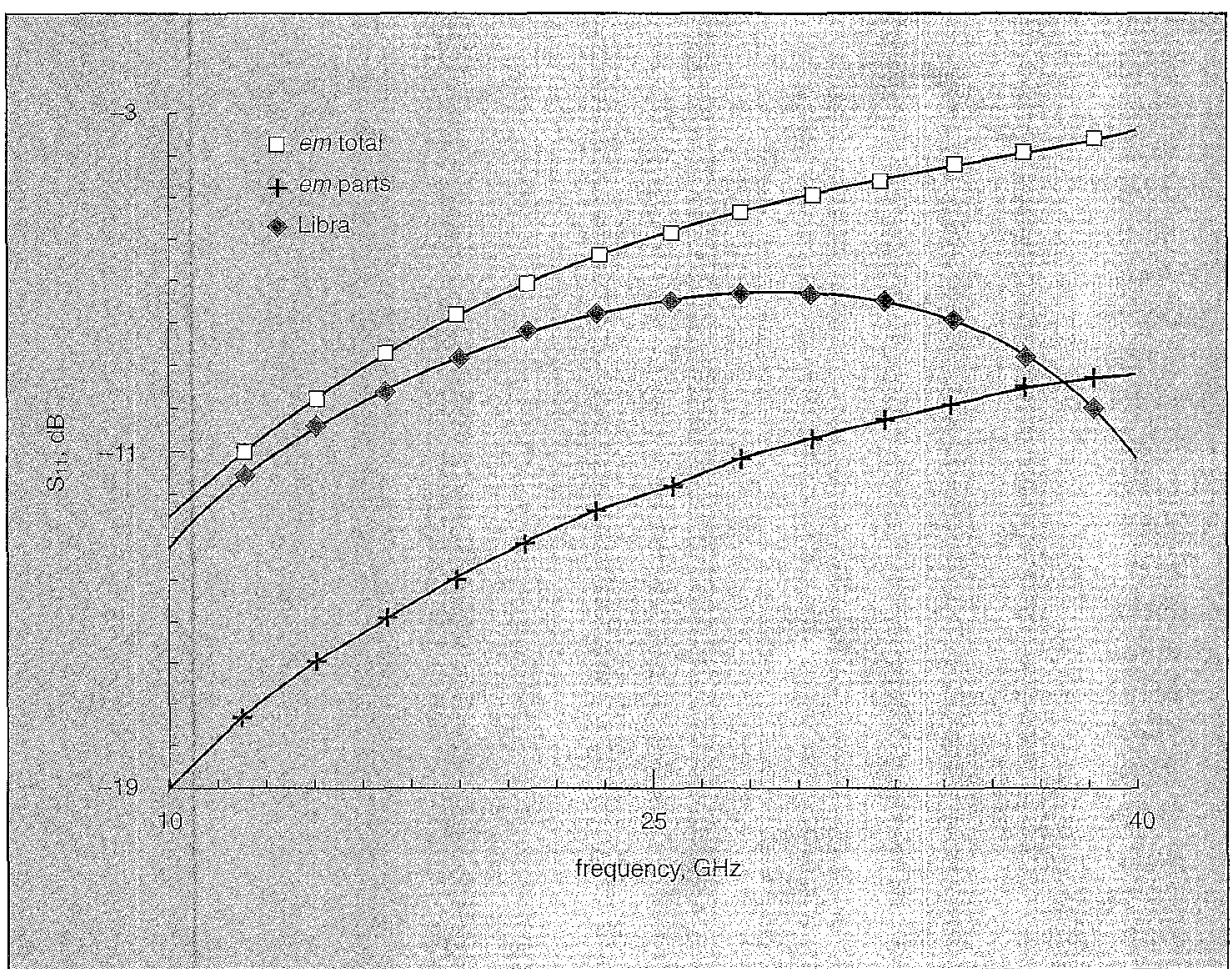

Fig. 9 Comparison between models for $S_{11}$ of meandered line domain : codes. The importance of time-domain field simulators in the future is emphasised with the increasing use of system simulation where digital components are included alongside RF components in many applications. For 3-D arbitrary electromagnetic simulators, a minimum 64 Mbytes of RAM is generally required, and at least 200 Mbytes swap space (a good rule is to keep 2.5 to 3 times the amount of RAM for swap space) is needed in the hard disk. Additional memory is recommended to increase the size and complexity of the models that can be analysed. Likewise, around 100 Mbytes are needed to load and store the program. frequency-domain codes is still debatable. Frequency domain codes (em, Maxwell-Strata, Maxwell-Eminence ${ }^{\mathrm{TM}}$, HFSS, Momentum, Ensemble ${ }^{\mathrm{TM}}$, Microwave Explorer, Mafia-E2/E3 ${ }^{\mathrm{TM}}$, etc) provide a point-to-point approach where some data is available while the simulation continues and are appropriate to obtain the boundary solutions for waveguide ports. ${ }^{18}$ Thus, it would seem desirable to use frequency-domain-based simulators rather than having to wait for all the results of a single run, especially for problems requiring high-frequency resolution. However, although time-domain simulations (Micro-Stripes, MicroWaveLab, MafiaT2/T3 ${ }^{\mathrm{Tm}}$, etc.) are computationally more demanding than frequencydomain codes (slower), the importance of time-domain electromagnetic simulation is increasing. According to Jansen, ${ }^{18}$ this is due to the maturing of time-domain electromagnetic algorithms and the further increase in computational power available at low cost. Timedomain simulators provide a much more physical insight in the circuit, and since they usually employ differentialbased equations, the memory requirements are lower than for frequency-

\section{Some examples}

The need for electromagnetic simulators is illustrated here with some examples. First, consider the meandered line on xgeom (Sonnet Software) shown in Fig. 8. The designer might expect that the parasitic coupling between the arms of the meandered line would degrade the final results. The Libra_3.0 model for the microstrip bends starts degrading at around $23 \mathrm{GHz}$, which is the maximum frequency given by the range of validity. This is because

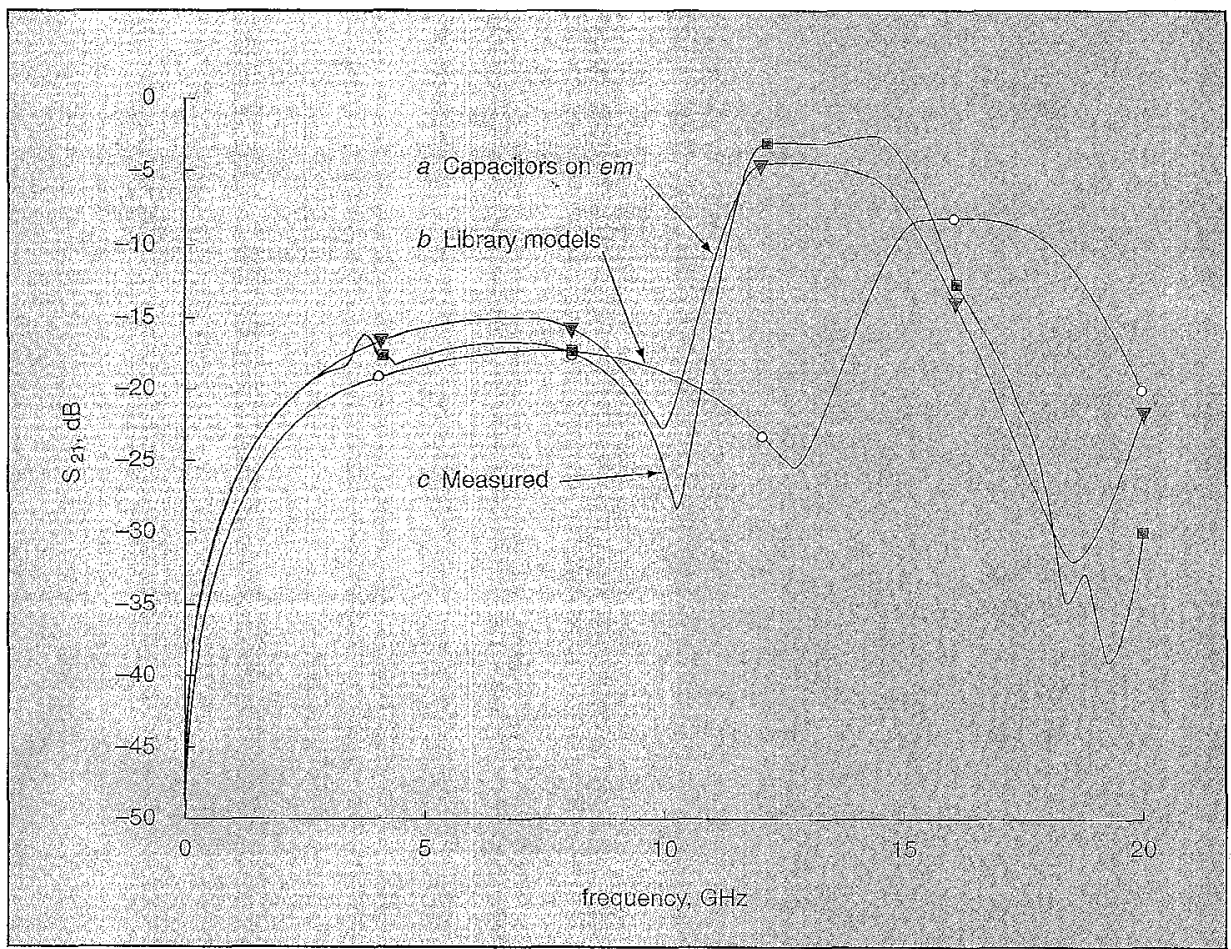

Fig. 10 Transmission response of MMIC ring: (a) simulated with capacitors modelled on em; (b) simulated with lumped capacitor models; (c) measured data 
these models were based upon a large number of test structures with widely varying microstrip widths but with a limited frequency range. A comparison between the Libra_3.0 model and two electromagnetic simulations, one using the so-called decomposition technique and one for the entire layout, is given in Fig. 9. This example clearly illustrates the importance of electromagnetic simulation.

Another interesting example of the extensive use of electromagnetic simulators on MMIC design is the monolithic ring filter of Fig. 7 . This filter ${ }^{19}$ was fabricated using an experimental multilayer MMIC process developed at King's College by Dr. Rezazadeh's team in the Physical Electronics Group. The simulation of the polyimide overlay capacitors is crucial to achieve accurate prediction of the transmission response, as depicted in the comparison of measured and modelled results shown in Fig. 10. Standard lumped capacitor models would have only two ports and would not accurately represent the distributed nature of the capacitor's plates.

\section{System simulation}

There is an increasing need for component-oriented methods which are easier to use for complex subsystems. System simulation programs treat individual circuits as black boxes with some kind of describing function (not just $S$-parameters) in order to predict overall system performance (gain, noise, dynamic range, nonlinearities, spurious signals, intermodulation distortion, etc.). This enables complex subsystems, such as complete transmitters and receivers, to be designed at a high level. MDS can analyse complex subsystems with its standard simulators: as an example, Fig. 11 shows an example of a QPSK transceiver system on MDS.

Since systems are becoming increasingly complex, and as digital signal processing becomes more closely integrated with microwave hardware, system level simulation is becoming more important and is receiving a great deal of attention. The Communications Design Suite by HP-EEsof currently represents the state-of-the-art in the field.

\section{Summary}

The distinct feature of microwave $\mathrm{CAD}$ is the need for a layout-oriented approach to circuit modelling. The key differences between Touchstone and SPICE are the microstrip library elements and the frequency-domain analysis. However, the use of equivalent circuit models for discontinuities is fundamentally flawed because important electromagnetic interactions are often neglected with this building-block approach. The demand for increased accuracy has led to microwave simulators which are based entirely on electromagnetic principles. In parallel, the need to accurately predict the performance of power amplifiers, mixers, etc. has led to the development of nonlinear simulation techniques which combine time-domain and frequency-domain methods.

Much of the impetus for the development of improved CAD has come from the MMIC industry: MMICs are expensive to fabricate and cannot readily be tweaked, so the design has to be right first time. The latest integrated MMIC CAD environments offer the ability to use a number of different types of simulation and component model within a single design file. Electromagnetic modelling and SMART library elements stand out as two particularly important developments for the MMIC designer. However, it is as important as ever for the designer to be aware of the limitations of the models that he is using. Furthermore, the treatment of component interactions (e.g. coupling between spiral inductors) is still limited.

This paper has touched on the subject of MMIC design and layout and SMART libraries. A further paper in this $\mathrm{CAD}$ series will describe the MMIC design and layout process in more detail.

\section{Acknowledgments}

The authors would like to acknowledge the financial support of the Engineering and Physical Sciences Research Council, and the Comision Interministerial de Ciencia y Technología (CICYT), Spain, under the project TIC95-0983-C03-02. We would like to thank Hewlett Packard, Barnard Microsystems, Sonnet Software, Optimization Systems Associates, Kimberley Communications Consultants and Ansoft Corporation for their generous educational discounting. The assistance of Dr. D. M. Brookbanks at GEC-Marconi Materials Technology Ltd. (Caswell) is gratefully acknowledged. 
San Rebertson bbtained BS

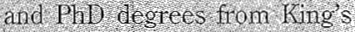

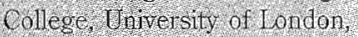
11.1 984 ait 1960 . espectively. 17ron 1684 to 1966 . We was eniployed at llessey Research (astivel) in: the : MMLC Research (G ouj, viere he worked on MVI rixers. on Wafer neas trement technidues ard It T (characterisaton. In 1986 he returned to Kun's Colleoc. hist as a Resurch

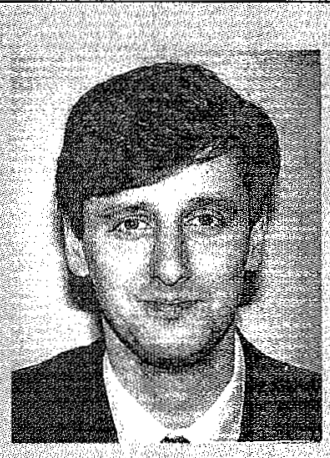
Assistant forking on the ISAM noble communitations

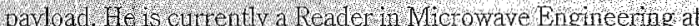
King S College and leader of the MMO Reseachleam th the

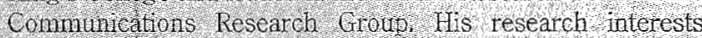
enconnass all is bects of the design and apphication of MMLS.

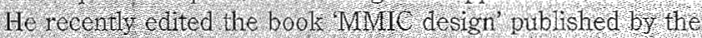

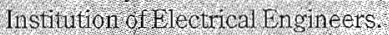

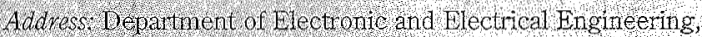

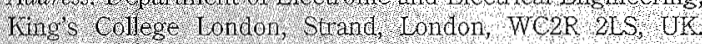

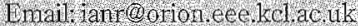

Mlun haracavoglu recerved?

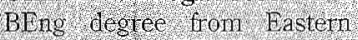
Mediter tanean V Triversiy oymus in 1991 and an MS.

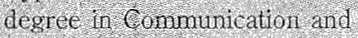

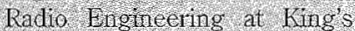

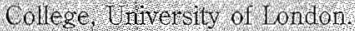

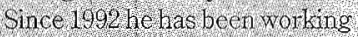
as a Researeh Assuciate in the MMIC Research. Iean at

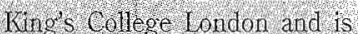
Misulns stuties towards the

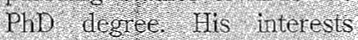

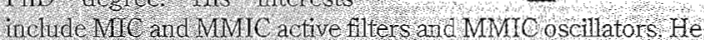

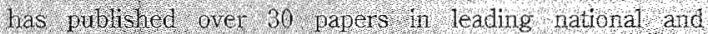

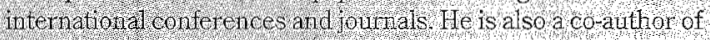

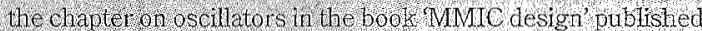

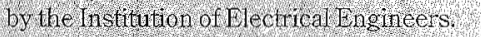

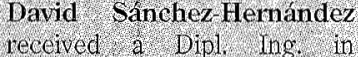
Teleconmivinicalions Engineer 119. 11011 . Untrersidad

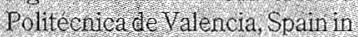
1992. 1.20n 1992 to 1904 he worled as d Research Assistant

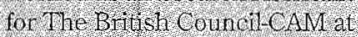
Ting:s: Vollege London. Siree 1991 le 1 tas beca a Resear.

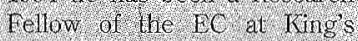
(. olle, se, where he las woulded on several vojects relaled 10

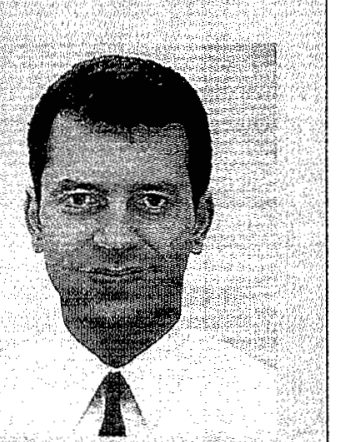

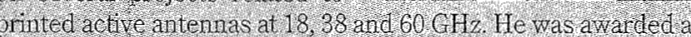
T11) 11996 tor researeh concentrated on dua band and actue

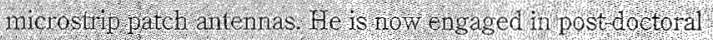

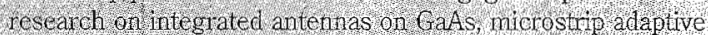

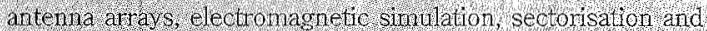
dversigted hives

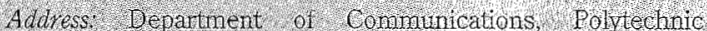

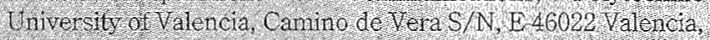

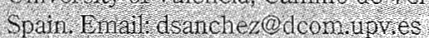

References

1 EDWARDS, T.C.: 'Foundations for microstrip circuit design' (John Wiley \& Sons, 1992, 2nd edn.)

2 GILIICK, M., ROBERTSON, I. D., and AGHVAMI, A. H.: 'Uniplanar techniques for MMICs', Electron. \& Commun. Eng. J., August 1994, 6, (4), pp.187-194

3 MATTHEI, G.L., YOUNG, L., and JONES, E.M.T.: 'Microwave filters, impedance-matching networks, and coupling structures' (McGraw-Hill, New York, 1964)

4 WHEELER, H.A.: 'Transmission-line properties of a strip on a dielectric sheet on a plane', IEEE Trans. on Microwave Theory \& Tech., 1977, 25, (8), pp. 631-647

5 HAMMERSTAD, E. O.: 'Equations for microstrip circuit design'. Proc. 5th European Microwave Conf., 1975, Hamburg, pp. 268-272

6 NAGEL, L. W., and PEDERSON, D. O.: 'Simulation Program with Integrated Circuit Emphasis'. Electronics Research Laboratory Rep. No. ERL-M382, University of California, Berkeley, 1973

7 HOEFER, W. J. R. et al.: 'Evaluation of the equivalent circuit parameters of microstrip discontinuities through perturbation of a resonant ring', IEEE Trans. on Microwave Theory \& Tech., 1975, 23, pp. 1067-1071

8 KIRSCHNING, M. et al.: 'Measurement and computer-aided modeling of microstrip discontinuities by an improved resonator method'. 1983 IEEE Microwave Theory \& Tech. Int. Symp. Digest, pp. 495-497

9 KOSTER, W., NORBERT, H. L., and JANSEN, R. H.: 'The microstrip step discontinuity: a revised description', IEEE Trans. on Microwave Theory \& Tech., 1986, 34, (2), pp. 213-223

10 HOFFMAN, R.: 'Handbook of microwave integrated circuits' (Artech House, 1987), pp. 267-309

11 HAMMERSTAD, E. O.: 'Computer-aided design of microstrip couplers using accurate discontinuity models'. 1981 IEEE Microwave Theory \& Tech. Int. Symp. Digest, pp. 54-56

12 KRYLOFF, N., and BOGDLIUBOFF, N.: Introduction to nonlinear mechanics' (Princeton University Press, Princeton, NJ, 1968)

13 KUNDERT, K. S., et al: 'Applying harmonic balance to almostperiodic circuits', IEEE Trans. on Microwave Theory \& Tech, 1988, 36, (2), pp. 366-377

14 HWANG, V.D., et al.: 'Nonlinear modeling and verification of MMIC amplifiers using the waveform-balance method', IEEE Trans. on Microwave Theory \& Tech., 1989,37,pp. 2125-2133

15 GILMORE, R. J., and STEER, M. B.: 'Nonlinear circuit analysis using the method of harmonic balance - a review of the art. Part I. Introductory concepts', Int. J. Microw. Millim.-Wave Comput.-Aided Eng., 1991, 1, (1), pp. 22-36

16 RAUTIO, I. C.: 'Some comments on electromagnetic dimensionality', IEEE Microwave Theory \& Tech. Soc. Newsletter, Winter 1992, pp. 23

17 BANDLER, J. W., et al:: 'Electromagnetic optimization exploiting aggressive space mapping', IEEE Trans. on Microwave Theory \& Tech., 1995, 43, (12), pp. 2874-2882

18 CAD Roundtable: 'Diverse views on the future of RF design', in Microwave engineering Europe directory 1996', pp. 20-26

19 WANG, Q. H., GOKDEMIR, T., BUDIMIR, D., KARACAOGLU, U., REZAZADEH, A. A., and ROBERTSON, I. D.: 'Fabrication and microwave characterisation of multilayer circuits for MMIC applications', IEE Proc., Microw. Antennas Propag., June 1996, 143, (3), pp. 225--232

(C) IEE: 1996

First received 4th April and in revised form 4th July 1996 